\title{
What do we know about the long-term cognitive and behavioral outcomes of school-aged children who were born moderate to late preterm?
}

\author{
Eun Sun Kim, MD, PhD \\ Department of Pediatrics, Kangwon National University School of Medicine, Chuncheon, Korea
}

With improvements in neonatal intensive care unit (NICU) care, major neurologic disabilities such as cerebral palsy, mental retardation, blindness, and deafness seem to have decreased. ${ }^{1-3)}$ However, the cognitive/behavioral outcomes of school-aged children who were born prematurely are worse than those of full-term children. A meta-analysis of school-aged children born prematurely showed that mean cognitive scores were significantly lower (weighted mean difference, 10.9; 95\% confidence interval, 9.2-12.5) than those of full-term children. ${ }^{4)}$ It is notable that intelligence quotient (IQ) scores show a gestational agerelated gradient, especially in children born before 33 weeks.5) In addition, most studies show an increased risk of attention problems such as attention-deficit/hyperactivity disorder (ADHD).,5) Some studies included executive function and academic achievement in school-aged children who were born prematurely, showing their deficits which lags behind full term-born peers. $\left.{ }^{6}\right)$

Those studies focus on very preterm ( $<32$-week gestation) or very low birth weight $(<1,500 \mathrm{~g})$ infants. Meanwhile, long-term cognitive and behavioral outcomes of moderate (32- to 33-week gestation) and late (34- to 36-week gestation) preterm infants are relatively unknown even though they account for $88 \%-89 \%$ of preterm births in Korea. In this issue of Clinical and Experimental Pediatrics, Jin et al. $\left.{ }^{7}\right)$ reported on the single-center long-term neurodevelopmental outcomes of children born moderate to late preterm; this is the first Korean report of a wide range of neurodevelopmental outcomes including cognition, executive function, and behavioral problems of school-aged former moderate to late preterm infants. The study found that about one quarter of the children had cognitive problems (IQ, 70-84) and more than half had abnormal scores on ADHD screening tests. They also found that about one quarter of the children had borderline scores in executive function tests, suggesting possible deficits in academic achievement. ${ }^{8)}$ As this large population of moderate to late preterm infants is sometimes underestimated or neglected during follow-up evaluations, the study suggests the importance of long-term follow-up of those infants.

The follow-up rate of the long-term outcome study is impor- tant because highly followed up data represent the group characteristics better. The study of Jin et al..$^{7)}$ described a relatively low follow-up rate and explained practical reasons for including such a small number of children in the methods section with a flow chart. In addition, the authors analyzed clinical characteristics between followed and nonfollowed populations and showed no difference, which partially resolves the selection bias.

Preterm longitudinal studies typically need term control groups. The most ideal control group is full-term siblings who contribute to the controlling of postnatal environmental factors. Classmate controls matched for age and sex can be also used as a control group. The control group should be evaluated at the same time point because the result of the study group can be developmental problems or cohort-specific problems. ${ }^{5)}$ Besides, IQ scores tend to drift upward over time and obsolete tests can underestimate if there is no contemporary control group. ${ }^{5)}$ As the study of Jin et al. ${ }^{7}$ ) mentioned as a limitation, they could not include a full-term control group. Instead, they supplemented data from the full term-born population in cognitive and executive function tests in their discussion.

One interesting point is that there was no significant association with perinatal/socioeconomic factors and neurocognitive results in this study. As severe neurologic deficit cases were excluded and no children in the study group had a severe brain injury, other perinatal factors showed no significant impact on long-term outcomes. Postnatal environmental factors such as parenting, education, or economic status are possible influential factors of neurocognitive outcomes in preterm infants. Parental intervention was suggested as a method of achieving better neurocognitive outcomes of preterm infants once they reach school age. ${ }^{9)}$ The study of Jin et al..$\left.^{7}\right)$ did not analyze parenting; however, they uncovered disadvantages in cognitive, executive, and behavioral outcomes of former moderate to late preterm children regardless of perinatal, economic, and maternal educational factors.

The study of Jin et al..$^{7}$ cohort did not show severe cognitive deficits $(\mathrm{IQ}<70)$; rather, they were comparable with very pre-

Corresponding author: Eun Sun Kim, MD, PhD. Department of Pediatrics, Kangwon National University School of Medicine, 1 Gangwondaehak-gil, Chuncheon 24341, Korea 
term children. However, highly prevalent (about a quarter) and low severity (borderline) cognitive problems should be em. phasized. Although abnormal scores in ADHD screening tests do not confirm an ADHD diagnosis, attention problems were highly prevalent in the cohort. Children with a borderline IQ or attention problems may show overlapping executive function deficits. As borderline or subtle neurodevelopmental problems are more responsive to early intervention, the developmental trajectory of moderate preterm and NICU-admitted late preterm infants should be closely followed through adolescence and even into adulthood. ${ }^{10)}$

In conclusion, moderate to late preterm infants are at risk of developing cognition, behavior, and executive function problems at a high prevalence during the school years. Periodic follow-up and population assessments are important. Further studies with high follow-up durations and any appropriate term control group are required to confirm the long-term outcomes of moderate to late preterm infants.

\section{Conflicts of Interest}

No potential conflicts of interest relevant to this article are reported.

See the article "Long-term cognitive, executive and behavioral outcomes of moderate and late preterm at school age" via https:// doi.org/10.3345/kjp.2019.00647.

\section{References}

1. Reid SM, Carlin JB, Reddihough DS. Rates of cerebral palsy in Victoria, Australia, 1970 to 2004: has there been a change? Dev Med Child Neurol 2011;53:907-12.

2. Pierrat V, Marchand-Martin L, Arnaud C, Kaminski M, Resche-Rigon M, Lebeaux C, et al. Neurodevelopmental outcome at 2 years for preterm children born at 22 to 34 weeks' gestation in France in 2011: EPIPAGE-2 cohort study. BMJ 2017;358:j3448.

3. Moore T, Hennessy EM, Myles J, Johnson SJ, Draper ES, Costeloe KL, et al. Neurological and developmental outcome in extremely preterm children born in England in 1995 and 2006: the EPICure studies. BMJ 2012;345:e7961.

4. Bhutta AT, Cleves MA, Casey PH, Cradock MM, Anand KJ. Cognitive and behavioral outcomes of school-aged children who were born preterm: a meta-analysis. JAMA 2002;288:728-37.

5. Johnson S. Cognitive and behavioural outcomes following very preterm birth. Semin Fetal Neonatal Med 2007;12:363-73.

6. Aarnoudse-Moens CS, Weisglas-Kuperus N, van Goudoever JB, Oosterlaan J. Meta-analysis of neurobehavioral outcomes in very preterm and/or very low birth weight children. Pediatrics 2009;124:717-28.

7. Jin JH, Yoon SW, Song J, Kim SW, Chung HJ. Long-term cognitive, executive and behavioral outcomes of moderate and late preterm at school age. Clin Exp Pediatr 2020 Feb 6 [Epub]. https://doi.org/10.3345/ kjp.2019.00647.

8. Alloway TP, Alloway RG. Investigating the predictive roles of working memory and IQ in academic attainment. J Exp Child Psychol 2010;106: 20-9.

9. Treyvaud K, Doyle LW, Lee KJ, Ure A, Inder TE, Hunt RW, et al. Parenting behavior at 2 years predicts school-age performance at 7 years in very preterm children. J Child Psychol Psychiatry 2016;57:814-21.

10. Vohr B. Long-term outcomes of moderately preterm, late preterm, and early term infants. Clin Perinatol 2013;40:739-51. 